\title{
Exploring vitiligo susceptibility and management: a brief review
}

\author{
Razia Rahman and Yasha Hasija*
}

\begin{abstract}
Background: Vitiligo is a common dermatological disorder of chronic depigmentation which is phenotypically characterized by white macules on the skin caused as a result of the systematic destruction of functional melanocytes. This review provides an overview of vitiligo, its etiopathogenesis and disease management, and also discusses the scope of network-interaction studies and polypharmacological studies in understanding vitiligo disease module.

Methods: A narrative review of the relevant published literatures known to the authors that comprehensively discussed about vitiligo and its implications was conducted.

Results: Emerging evidence underlines the existing connection between deregulated miRNA function and vitiligo pathogenesis. It has also been linked with autoimmunity for the cause of melanocyte death in susceptible individuals. Alteration of genetic factors involved in immune responses and melanogenesis along with environmental factors are central to disease manifestation. Screening methods as such are not available for vitiligo, and the diagnosis is based on the assessment of the absence of melanocytes from the lesions in the affected area. With the occurrence of vitiligo at any age, most people typically develop it at a young age. Depending on the disease course and duration, clinical management primarily involves disease stabilization either by repigmentation or depigmentation of the skin.

Conclusions: Several questions remain unsolved which indeed makes vitiligo an excellent model for studying autoimmune and degenerative processes. An understanding of the underlying degenerative mechanisms and unraveling the biological mediators of melanocyte loss will open up avenues for testing novel therapeutic approaches in vitiligo management. Such studies can revolutionize our apprehension of the molecular interconnections that underpin vitiligo pathogenesis.
\end{abstract}

Keywords: Vitiligo, Autoimmunity, Genetics, Disease management

\section{Background}

Evolutionary studies indicated that skin pigmentation was the result of adaptive responses to the environment (UV radiation) after the loss of hair coat in humans. Pigmentation provides photoprotection and participates in skin barrier function and antimicrobial defenses of the skin, hence, is essential for maintaining body homeostasis (Jablonski and Chaplin 2010). Dermatological disorders have a significant psychosocial impact on the quality of patients' lives since their symptoms are visible. Although dermatological conditions are not life-threatening, it is the product of this morbidity multiplied by the high prevalence of skin disease which results in a large burden of the disease in absolute

\footnotetext{
* Correspondence: yashahasija06@gmail.com

Department of Biotechnology, Delhi Technological University, Shahbad Daulatpur, Main Bawana Road, Delhi 110042, India
}

terms. Vitiligo is one such dermatological disorder that is inflicted upon almost all age groups irrespective of gender and skin type. Vitiligo is a complex acquired depigmentation disorder characterized by white non-scaly patches with distinct sharp margins distributed unilaterally in the skin. It results in the episodically selective disappearance of functional melanocytes which leads to pigment dilution in the affected areas (Le Poole et al. 1993; Ezzedine et al. 2012a, b). Vitiligo is usually asymptomatic and can appear anywhere on the skin commonly affecting the areas of the face and hands, and the genitals (Ezzedine et al. 2012a, b). It might also lead to whitening of hair although the skin and hair are affected to different degrees depending on the disease course which is unpredictable in most cases with phases of stabilized depigmentation eventually with the duration of the disease (Picardo et al. 2015).

(c) The Author(s). 2018 Open Access This article is distributed under the terms of the Creative Commons Attribution 4.0 International License (http://creativecommons.org/licenses/by/4.0/), which permits unrestricted use, distribution, and 
The onset of vitiligo and its subsequent progression is thought to be impelled by several inherited genes and can develop at any age irrespective of the type of skin, gender, race, or geographical location. Additional stochastic or environmental factors are also likely to further influence the pathogenesis of vitiligo (Spritz 2013). It affects the life of patients both biologically and psychologically due to its unsightly appearance inflicting significant psychological stress and exerting a pernicious influence on the quality of life in patients concerning self-esteem and social interactions. The development of new lesions resulting in enlarged macules is classified as an active form of the disease that warrants more aggressive therapy (Taieb 2012). Friction has also been indicated to trigger vitiligo development in areas such as the neck, elbows, and ankles, a phenomenon known as the Koebner phenomenon (Gauthier et al. 2003). It has been proposed that in some vitiligo cases, Koebner phenomenon could be used to predict and analyze the clinical profile and disease course (van Geel et al. 2012). Various genetic, experimental, and clinical studies have unraveled significant pathways in vitiligo pathogenesis that have helped in identifying targets for the development of efficient treatment approaches for disease management.

\section{Epidemiology and prevalence}

Vitiligo is the most common depigmentation disorder with an overall estimated worldwide prevalence of 0.5 to 2\% (Krüger and Schallreuter 2012) but a higher prevalence rate up to $8.8 \%$ has been reported in India (Sehgal and Srivastava 2007). It has also been reported to occur at an early age in the women population. The largest epidemiological study of the prevalence of vitiligo was carried out on 47,033 inhabitants on the island of Bornholm in Denmark in 1977, where vitiligo was found to affect $0.4 \%$ of the population (Howitz et al. 1977). Similar results were obtained in French West Indies, predominantly in the black population (Boisseau-Garsaud et al. 2000). Reports from China, Mexico, and Japan also indicate high incidences of vitiligo with an overall prevalence rate of $0.6 \%$ (Sehgal and Srivastava 2007; Wang et al. 2013).

The vitiligo prevalence rates of 0.06 to $2.3 \%$ in the general population and 0 to $2.2 \%$ in children have been reported in earlier studies (Nicolaidou et al. 2012). Findings also indicate an increased rate of prevalence with respect to age (Alikhan et al. 2011) (Table 1), increasing from $0.5 \%$ in children of less than 1 year of age to $1 \%$ in children of $1-5$ years of age and further expanding to $2.1 \%$ in 5-12 year olds. The onset of vitiligo before the age of 12 years is reported to range from 0-2.6\% (Halder 1997; Nicolaidou et al. 2012). In almost half of the patients, vitiligo develops before the age of 20 years with nearly about 70 to $80 \%$ developing it before 30 years of age. Segmental vitiligo also tends to occur at a younger age with $87 \%$ of cases reported in patients before 30 years of age and $41.3 \%$ cases before 10 years of age
Table 1 Increased prevalence rate of vitiligo with respect to age

\begin{tabular}{ll}
\hline Prevalence rates & Percentage \\
General population & $0.06-2.3 \%$ \\
Children & $0-2.2 \%$ \\
Of less than 1 year & $0.5 \%$ \\
$1-5$ years of age & $1 \%$ \\
$5-12$ year olds & $2.1 \%$ \\
Before the age of 12 years & $0-2.6 \%$ \\
Onset of disease & Percentage \\
Before 20 years of age & $50 \%$ \\
Before 30 years of age & $70-80 \%$ \\
\hline
\end{tabular}

(Ezzedine et al. 2012a, b). Discrepancies between prevalence and incidence data leads to variability in epidemiological data which is attributable to the diverse populations and its genetics, differences in disease classification, cultural and social differences, lack of screening or diagnostic tests, social and cultural stigma attached to the disease, and inconsistent reporting by patients. Despite the same prevalence ratio of $1: 1$ in males and females, the ratio of treatment requests is 1:2. This signifies that females seek treatments more frequently, which apparently might be due to the ramifications of greater social burden (Alikhan et al. 2011).

\section{Classification of vitiligo}

Figure 1 shows the major classifications of vitiligo, namely, non-segmental or generalized vitiligo, segmental vitiligo, and mixed vitiligo according to international consensus (Ezzedine et al. 2012a, b). The onset of mixed vitiligo is the same as segmental vitiligo which eventually develops into non-segmental vitiligo, thus, the name mixed vitiligo.

In non-segmental (generalized) vitiligo, which is the most common form of vitiligo, the depigmented patches develop on both sides of the body and usually progresses slowly (Picardo et al. 2015). While in segmental vitiligo, the patches are limited to only one side of the body, particularly in the face and trunk area, and do not usually cross the midline of the body. It initially progresses rapidly which spontaneously stabilizes after a period of 6 months (Ezzedine et al. 2012a, b). In segmental vitiligo, typical distribution patterns on the face and trunk have been described, which aid in differential diagnosis (Kim et al. 2011; Geel et al. 2014). Mixed vitiligo, on the other hand, has been described as a rare combination of both segmental vitiligo and non-segmental vitiligo. The onset of mixed vitiligo is the same as segmental vitiligo which eventually develops into non-segmental vitiligo, thus the name mixed vitiligo (Ezzedine et al. 2011). The markedly different distribution patterns aid in recognition of the type of vitiligo as the evolution and the kind of treatment are different for the different subtypes of vitiligo. 


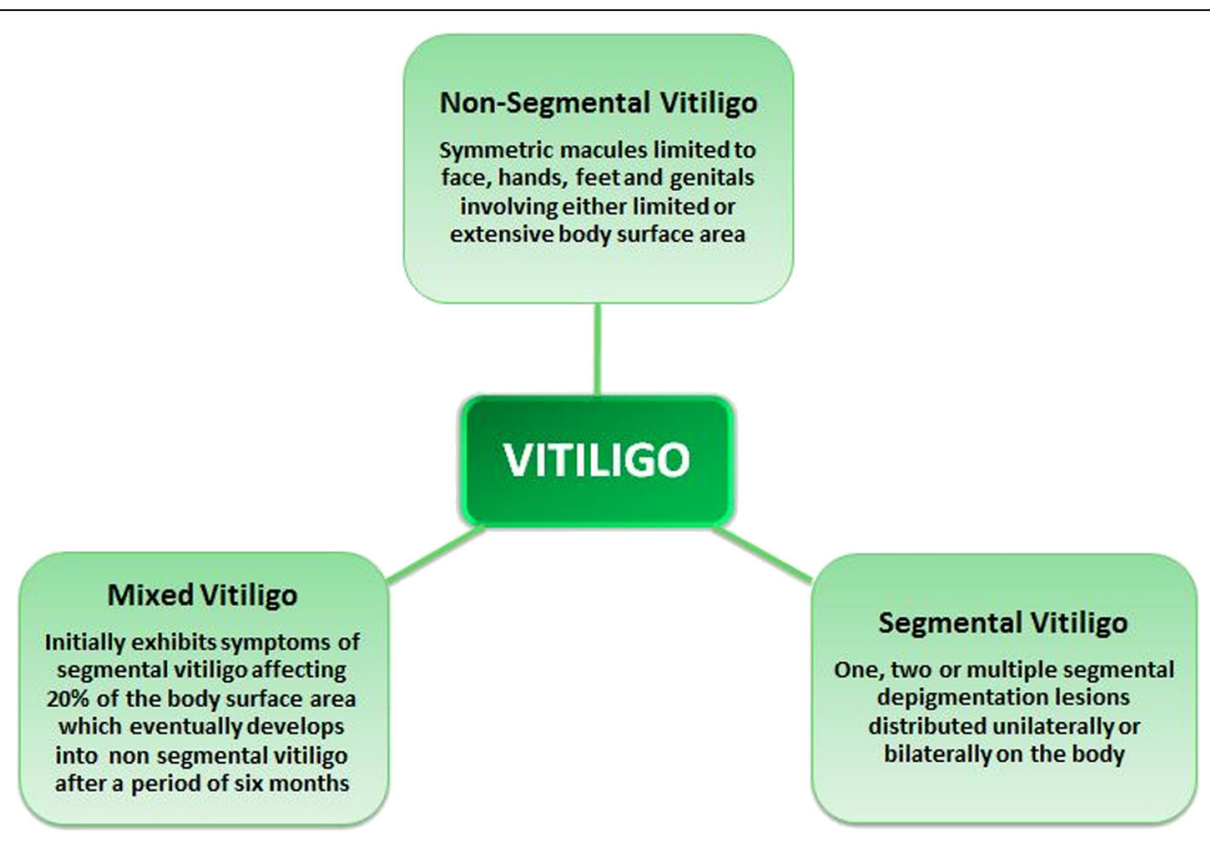

Fig. 1 Classification of vitiligo

\section{Etiopathogenesis of vitiligo}

Stress responses in the skin eliciting an autoimmune response in genetically susceptible individuals are thought to be instigated by a trigger event that eventually targets the melanocytes predisposing individuals to develop vitiligo. Though recent studies have started to reveal the etiopathogenesis of vitiligo, the mechanisms leading to vitiligo are yet a debatable topic. However, several hypotheses have been presented signifying its association with the development of vitiligo (Fig. 2). Among the different theories developed, namely, autoimmunity, oxidative stress, melanocyte growth and defective melanocyte adhesion, viral infections, and neural mechanisms, the autoimmune theory is currently considered and accepted

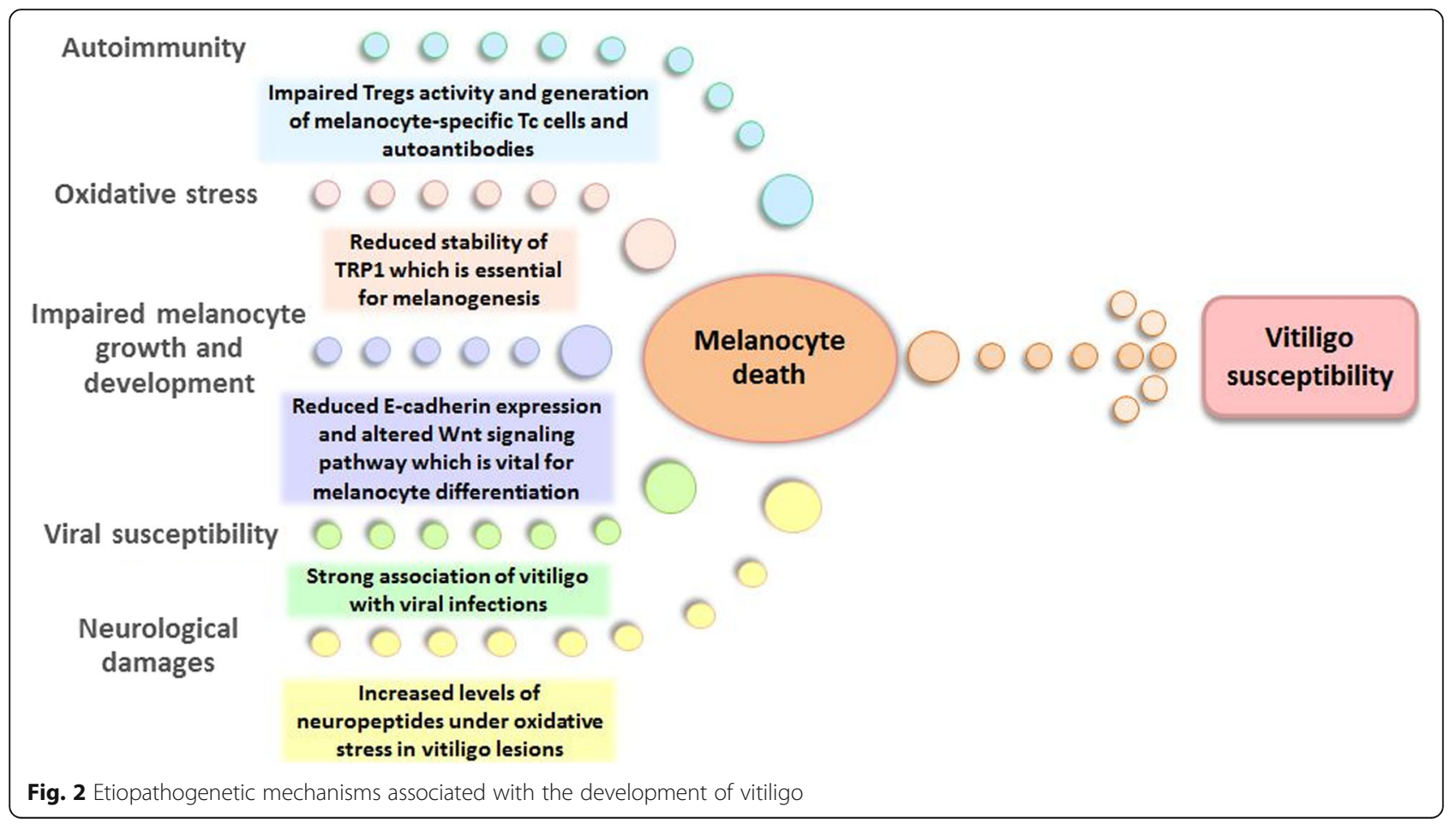


as the leading theory globally, which has been substantiated by several reports on the frequent association of vitiligo with autoimmune diseases (Rezaei et al. 2007; Le Poole and Luiten, 2008; Kasumagic-Halilovic et al. 2013; Strassner and Harris 2016). Also, the association of vitiligo with halo naevus, which is characterized by depigmented halo-like area circumscribing a mole encompassing dense immune cell infiltrate, further supports the importance of immune mechanisms in vitiligo development (Spritz 2010).

\section{Autoimmune theory}

The loss of self-tolerance in the pathogenesis of vitiligo is unclear and not yet well understood. High levels of circulating melanocyte autoantibodies recognized by $\mathrm{T}$ cells specifically against tyrosinase (TRP-1 and TRP-2) have been found in many vitiligo patients with their role being linked to the destruction of keratinocytes and melanocytes (Jimbow et al. 2001; Kemp et al. 2011). Autoimmunity in vitiligo has been suggested to develop owing to the failure of inherent mechanisms which are intended to control melanocyte proliferation (Le Poole and Luiten 2008). Other antigenic proteins related to disease activity, namely, glycoprotein 100 (gp100) and melanoma antigen recognized by $\mathrm{T}$ cells 1 (MART-1) have also been detected in the blood and tissues of vitiligo patients (Lang et al. 2001). Several studies have shown the accumulation of $\mathrm{T}$ helper $(\mathrm{TH})$ and $\mathrm{T}$ cytotoxic (TC) cells suggesting the formation of a silent micro-inflammatory process that kills melanocytes in the junction of the dermal and epidermal area of vitiligo lesion implying cell-mediated immune response activity (Oyarbide-Valencia et al. 2006). Certain major histocompatibility complex (MHC) alleles have been suggested to be associated with vitiligo as a vital link between the disease etiology and the aberrant self-antigen presentation to the $\mathrm{T}$ cells. Moreover, human leukocyte antigen (HLA)-A2 restricted, melanocyte-specific CD8+ T lymphocytes identified to kill melanocytes in the perilesional skin, has been detected in vitiligo patients (Van Den Boorn et al. 2009; Spritz 2010). Also, the fundamental role of regulatory $\mathrm{T}$ cells (Tregs) in the pathogenesis of vitiligo has been implicated in several reports with a reduction in their number in the peripheral blood of vitiligo patients along with their dysfunctional activity (Ben Ahmed et al. 2012; Lili et al. 2012). Also considered as a Th1-related disease, a significant increase in the concentration of the cytokines, namely, TNF- $\alpha$, IFNG, IL-10, IL1B, and IL-17 has also been reported to be associated with the onset as well as persistence of vitiligo in patients (Taher et al. 2009; Levandowski et al. 2013). Therefore, vitiligo serves as an eminent disease model to understand the initiation and progression of organ-specific autoimmune diseases.

\section{Oxidative stress theory}

Oxidative stress, which is the result of an increase in the levels of reactive oxygen species (ROS) and subsequent reduction of antioxidant enzymes, compromises the function of cellular proteins and membrane lipids, thus impairing the activity of the antioxidant system in both lesional and non-lesional skin (Maresca et al. 1997). This imbalanced status of the antioxidant system in vitiligo has been indicated to cause increased sensitivity of melanocytes to oxidative stress leading to cellular death. Excess levels of ROS have been reported in active vitiligo skin suggesting oxidative stress as the plausible cause of vitiligo pathogenesis (Jimbow et al. 2001). Superoxide dismutase, an antioxidant enzyme, has been reported to be altered in vitiligo skin indicating that ROS generation causes an alteration in the expression of the antioxidant system affecting melanocyte function (Sravani et al. 2009). Oxidative stress-driven reduction of TRP1 expression triggers the production of intermediates of toxic melanin leading to subsequent immune-mediated melanocyte destruction (Dell'anna and Picardo 2006). Experimental data revealed a close association between oxidative stress and immune responses that promote intrinsic damage. Histological analysis shows the expression of NLR family pyrin domain containing 1 (NLRP1), IL-1, and catalase (CAT) in developing lesions (Marie et al. 2014). Several polymorphisms have been reported in the CAT gene, which impairs the enzyme function of X-box binding protein 1 (XBP1) which is primarily involved in mitigating stress-induced inflammation (Wood et al. 2008). These factors are involved in stress responses which ultimately triggers innate immune responses.

\section{Deficient melanocyte adhesion and melanocyte growth theory}

Evidence of the decreased adhesive property of melanocytes in vitiligo has been reported in earlier studies (Gauthier et al. 2003). Reduced expression levels of E-cadherin have been observed in melanocytes prior to depigmentation development in vitiligo skin. During oxidative or mechanical stress, an altered E-cadherin expression incites the loss of adhesion in epidermal melanocytes due to the increased levels of anti-adhesion molecule, tenascin (Le Poole et al. 1997; Wagner et al. 2015). Loss of melanocytes from the epidermal layer due to deficient adhesion of melanocytes could be an early phenomenon in vitiligo. Also, alteration in the factors influencing successful differentiation and proliferation of melanocytes due to oxidative stress, such as altered Wnt signaling, may also render susceptibility to vitiligo.

\section{Viral theory}

Several studies have depicted a strong association between vitiligo and hepatitis $\mathrm{C}$ virus (HCV) and hepatitis $B$ virus (HBV) infections in vitiligo patients (Akbayir et al. 2004; 
Akcan et al. 2006). Also, the association of cytomegalovirus (CMV) infection with vitiligo was also suggested to provoke the deterioration of skin conditions in vitiligo (Toker et al. 2007). Furthermore, the suspicious association of herpes virus and the human immunodeficiency virus (HIV) infection with vitiligo has also been reported (Niamba et al. 2007).

\section{Neuronal mechanisms theory}

Clinical observations addressing the correlation of local neurological damage to skin depigmentation (whitening) suggest that neuronal mechanisms do have a role to play in vitiligo pathogenesis. Current evidence of the detection of neuropeptides in vitiligo lesions supports the neural hypothesis which might be the effect of inflammation rather than a triggering factor. An increased level of neuropeptides such as neuropeptide Y (NPY) has been observed in the marginal areas of vitiligo lesions triggered under the conditions of oxidative stress that is thought as a reason for the induction of vitiligo (Lazarova et al. 2000).

\section{Diagnosis and screening}

The diagnosis of vitiligo does not require confirmatory laboratory tests in the majority of cases irrespective of its clinical subtype. The absence of melanocytes from lesions can be assessed by in vivo confocal microscopy (non-invasive) or a skin biopsy with the use of specific markers. There are no screening methods available for vitiligo (Ezzedine et al. 2012a, b). The clinical screening of associated disorders on the diagnosis of vitiligo is currently a debatable topic. However, female patients and those with longer disease duration and involving greater body surface area are more likely to have autoimmune thyroid disease. Therefore, proper thyroid function and the presence of anti-thyroglobulin and anti-thyroid peroxidase antibodies need to be checked regularly in vitiligo patients (Gey et al. 2013).

\section{Therapeutic options and management of vitiligo}

The management of vitiligo becomes challenging considering its complex etiopathogenesis. There is no definite cure available for vitiligo. Therefore, the current optimal management options according to the recent consensus guidelines involves a personalized approach with the therapeutic choice influenced by several factors such as disease course and its impact, skin type, age, gender, age, affected area and its extent, and social and cultural life influences (Taieb 2012). Also, general measures of avoiding factors of mechanical stress, Koebner phenomenon and UV-exposure might be helpful in limiting depigmentation conditions (van Geel et al. 2012). Figure 3 shows a flowchart depicting the clinical management of vitiligo depending on the disease course and treatment outcomes.
Topical corticosteroids, immunomodulators, and antioxidants

Topical corticosteroids being the first-line of treatment option manage disease progression by initiating anti-inflammatory responses with trivial outcomes. Although repigmentation is observed in the face and neck, the trunk area and the extremities show limited repigmentation. Oral corticosteroid involving moderate dosage of corticosteroids (mini-pulse therapy) is used to arrest disease progression in active disease conditions where the repigmentation outcomes are rare (Njoo et al. 1998). However, the associated side effects oral corticosteroids limit its long-term use.

Topical immunomodulators such as tacrolimus and pimecrolimus attenuate the production of proinflammatory cytokines by inhibiting $\mathrm{T}$ cell activity, thereby enhancing melanocyte migration and pigmentation in vitiligo patients (Ormerod 2005). Similar to corticosteroids, the results mostly show repigmentation in the face with moderate effects at other sites of the body.

Although, according to the current consensus guidelines, the use of topical antioxidants is not recommended, however, they are frequently prescribed in relatively limited trials (Leone and Paro 2015). The use of oral antioxidants in combination therapy is sometimes considered in patients undergoing phototherapy.

\section{Phototherapy}

Narrow-band UVB (NB-UVB) phototherapy is an effective treatment choice that has long been recognized to induce repigmentation. The majority of the patients are observed to develop the signs of repigmentation with phototherapy. Topical treatments are also advised after completed phototherapy sessions to prevent recurrence of depigmentation (Sitek et al. 2007).

Photochemotherapy is also an option, but the recurrent side effects often accompany carcinogenic risk along with limited successful outcomes which restrict its use over NB-UVB where such risks are less evident (Bhatnagar et al. 2007).

\section{Surgery}

Pigment cell transplantation techniques such as cellular and tissue graft transplantation may offer a valuable alternative treatment option. The necessity of the disease stability, which is the primary criteria linked to successful outcomes, limits this treatment option to selective patients only (van Geel et al. 2010). It is effective in both stable non-segmental and segmental vitiligo patients. Regardless of the technique used, the stability of the lesions is a major criterion related to the outcome of the procedure.

\section{Combination therapy}

The complexity of the disease makes it necessary to use a combination of different treatments to address both 


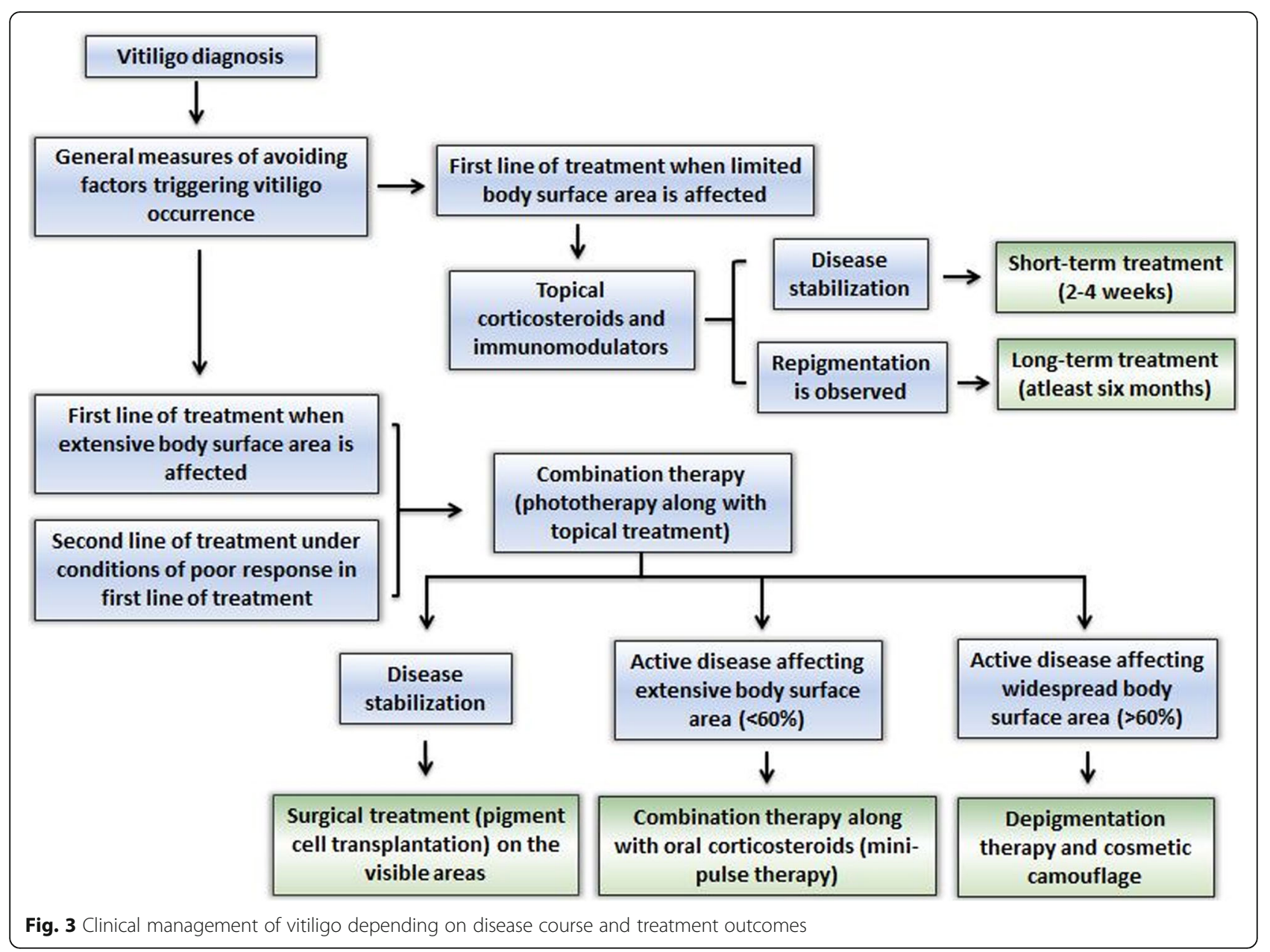

inflammatory responses on melanocyte differentiation and proliferation. Currently, NB-UVB is prescribed along with topical corticosteroids and immunomodulators, instead of its use as a monotherapy. Such combination therapies have been shown to accelerate the repigmentation rates (Nordal et al. 2011). However, the risk of skin cancer due to the combined use of immunosuppressants is a topic of both concern and debate.

\section{Depigmentation and cosmetic camouflage}

In conditions of extensive vitiligo where most parts of the body are affected by depigmentation, depigmenting the remaining pigmented areas is considered as a better option instead of repigmentation treatments. Bleaching creams, laser therapy, and cryotherapy are some of the options for depigmentation therapy (AlGhamdi and Kumar 2011). Regardless of this treatment method, repigmentation on the treated depigmented areas might occur and as such a permanent cure cannot be assured.

Camouflaging the depigmented areas with cosmetic products could aid to reduce the daily impact of the disease on social life (Hossain et al. 2016). However, specialized advice is required to be taken before going for such alternatives of cosmetic exposure to vitiligo skin.

\section{Association with other diseases}

Due to the polygenic nature of vitiligo, it is frequently associated with several autoimmune or autoinflammatory diseases, namely, thyroid disorders (Kasumagic-Halilovic et al. 2011), psoriasis, atopic dermatitis (Ezzedine et al. 2012a, b), diabetes mellitus, pernicious anemia, and Addison's disease (Rezaei et al. 2007). Antibodies directed against melanocytes and other organ-specific tissues have been found in vitiligo patients. Recent observations strongly point to vitiligo as an autoimmune disease sharing genes with other autoimmune disorders (Zhang and Xiang 2014). Recent studies reported the expression of markers on vitiligo melanocytes that have been detected in cells from neurodegenerative diseases, such as Alzheimer's (Bellei et al. 2013). Therefore, vitiligo might represent a degenerative disease model as well. Although the definite link between vitiligo and melanoma has not been fully elucidated yet, it has been reported that they both share an inverse relationship meaning that vitiligo-affected people are relatively protected 
having a much lower risk of developing melanoma' and vice-versa (Spritz 2010). Melanoma patients experiencing successful treatment responses were observed to consequently develop vitiligo. Both melanoma and vitiligo share CD8+ T cell antigens, and the allelic variants associated with vitiligo were found to confer protection from melanoma directly (Paradisi et al. 2014). This observation has contributed significantly to the development of improved therapeutic interventions to regulate melanoma in patients and depicts the similarity in the generation of immune responses and the target of similar antigens in both vitiligo and melanoma.

\section{Impact on the quality of life}

The implications of disease progression influence the potential psychiatric comorbidity associated with vitiligo. Skin integrity is considered relevant in many cultural and religious contexts, and any modification in the color or aspect of the skin influences the physical functioning and psychological state of the affected ones. Vitiligo has a major impact on the quality of patients' life relating to social and cultural stigma that eventually affects self-esteem and self-concept due to negative evaluation by others. Several factors including the patients' age at the onset of disease, the extent and distribution of the depigmented patches in the body, and stigmatization causing psychological trauma have a considerably influences the impact that vitiligo has on its patients. Furthermore, the discrepancies prevailing between the efficiency of treatment strategy, therapy response evaluation, and expectations of the patient and overall clinical management further add to the consternation of both the patients and their families (Kruger and Schallreuter 2013). Stigmatization has been shown to be the most influential factor in patient well-being and perceived quality of life, even more than disease duration and treatment inefficiency. In most cases, patients have reported discomfort owing to the uncontrolled progression of the disease rather than noting the development of new lesions in visible areas (Teovska Mitrevska et al. 2012). This suggests that the impaired quality of life is linked to the activity of the disease at a greater extent rather than to the involvement of exposed areas well indicating that the presence of visible lesions did not much affect the global pattern.

The onset of vitiligo in adolescence can have a long-lasting impact on a child's self-esteem directly affecting their psychological state causing interaction anxiousness and depression and thus pose as a risk factor for impaired quality of life in such children. Vitiligo has been found to cause more embarrassment and self-consciousness as children with vitiligo grow older with almost $95 \%$ of the teenagers within the age group of 15 to 17 years to be tribulated by their condition as compared to children (50\%) of age group between 6 to 14 years (Parsad et al. 2013).
Stress and anxiety caused by vitiligo being a precipitating factor for considerable psychosocial stress can be mitigated by self-help cognitive behavioral therapy (Shah et al. 2014). Treatment according to disease severity might not alone address a patient's suffering adequately. Increased awareness of the quality of life impairments is necessary to address the physical and psychological state that encompasses several factors from the patient's perspective and will help to assure that vitiligo patients are not under-treated and under-appreciated. More than a third of vitiligo patients experience symptoms of depression without necessarily fulfilling the criteria for clinical depression (Parsad et al. 2013). New vitiligo-specific impact scales that better reflects the burden caused by vitiligo have been recently developed such as VitQoL (Lilly et al. 2013), Vitiligo Life Quality (Senol et al. 2013), and Vitiligo Impact Patient Scale (Gupta et al. 2014; Salzes et al. 2016) since the high rate of depressive symptoms signifying the psychological impact in vitiligo patients is evident.

\section{Role of miRNAs and genetic variants on vitiligo susceptibility}

Vitiligo appears to be a multifaceted disorder underlying both genetic and non-genetic factors in a complex interactive manner. A comprehensive understanding of the molecular mechanisms that determine disease susceptibility, its onset, and phenotypic expression remains a challenge since the entire spectrum of this disorder is not yet clearly understood. Recent advances in genetic studies have led to the considerable progress in defining the genetic epidemiology and pathogenesis of vitiligo, and its relationships to other autoimmune diseases offering a real insight into its biological framework (Spritz 2013). These studies have improved our knowledge on vitiligo pathogenesis and have opened up new avenues for novel targeted therapies for lack of melanocyte regeneration in vitiligo leading to effective treatment approach as well as disease prevention.

\section{miRNA signatures}

MicroRNAs are conserved, small, endogenous non-coding RNA molecules that regulate post-transcriptional gene expression and promote translational repression leading to the cleavage and degradation of mRNA. Recent studies have shown that miRNAs play prime roles in various cellular, regulatory, and signaling processes maintaining physiological homeostasis. They are also essential for cellular morphogenesis, and any disruption in its architecture leads to disease development and progression. Serum miRNA expression profiles have been observed in vitiligo patients (Shi et al. 2013). Moreover, deregulated miRNA metabolism has been reported to be indicative of inflammatory skin conditions and linked to vitiligo pathogenesis (Mansuri et al. 2016). At present, characterization of interpretative miRNA expression 
and function in human melanocytes has elucidated them to be promising biomarkers for disease prognosis.

The collective role of miRNAs in oxidative stress and autoimmunity results in melanocyte destruction and leads to further progression of the disease. The expression levels of miR-1, miR-135a, and miR-9 targeting sirtuin 1 (SIRT1) which regulates stress response and inflammation have been found to increase in response to oxidative stress (Saunders et al. 2010), while heme oxygenase 1 (HO1) mRNA targeted by miR-183 is a stress-responsive antioxidant and anti-inflammatory factor (Chang et al. 2011). Increased expression of these miRNAs has been found in vitiligo patients suggesting their potential role in the destruction of melanocyte and formation of inflammatory micro-environment in the skin lesions caused as a result of oxidative stress. Increased levels of miR-133b expression have been observed in the lesional skin of vitiligo patients that suggests its role in the pathogenesis of vitiligo (Mansuri et al. 2014). let-7c targets the 3' UTR of IL10 and regulates its expression which is a pleiotropic cytokine possessing both anti-inflammatory and immunosuppressive properties (Jiang et al. 2012). A decrease in the level of IL10 expression has been noted in skin biopsies, peripheral blood mononuclear cells, and sera obtained from vitiligo patients well indicating towards its contribution to disease development (Ratsep et al. 2008). An increased expression of miR-99b, miR-125b, miR-155, and miR-199a-3p has also been reported in vitiligo-affected skin which is related to the inhibition of expression of melanogenesis-associated genes which demonstrates that the expression of these miRNAs are dysregulated in the skin of patients with vitiligo and suggests its contribution to vitiligo pathogenesis (Sahmatova et al. 2016). Another miRNA, miR-30b targets platelet-derived growth factor receptor $\beta$ (PDGFRB) mRNA and represses its ability to regulate cell proliferation in melanocytes resulting in melanocyte death in vitiligo patients (Shi et al. 2013). A significant increase in miR-183, miR-30a-3p, and miR-487a expression have also been shown in the lesional skin of vitiligo patients suggesting the importance of miR-183, miR-30a-3p, and miR-487a expression in rendering vitiligo susceptibility (Ruksha et al. 2017). Apart from the upregulated expression of miRNAs being responsible for vitiligo disease pathogenesis, individual miRNAs have been found to be significantly downregulated in the lesional skin as compared to controls. A downregulated miR-145 can restrict melanocyte proliferation and cause melanocyte death by inducing apoptosis via caspase- 3 and caspase- 7 activation, thereby playing a prime role in disease initiation (Sahmatova et al. 2016). The expressions of miR-211, miR-141, miR-136, miR-296, and miR-328 have been found to be specifically downregulated in vitiligo skin exhibiting their potential role in the development and progression of the disease (Mansuri et al. 2016).

\section{Genetic susceptibility}

In the recent years, several genome-wide association and linkage studies have been conducted in vitiligo which is a polygenic disorder with a complex mechanism of pathogenesis. Although multiple studies have demonstrated the genetic background of vitiligo development, however, the genetic risk is not absolute. Strong evidence for genetic factors in the pathogenesis of generalized vitiligo from various gene expression and association studies identified candidate genes. These candidate genes were found to be involved in encoding components of biological networks that primarily regulate the elements of the immune system and their targeted destruction of melanocytes mediating vitiligo susceptibility (Spritz 2012). Accordingly, several potent disease-contributing loci has been identified to be associated with vitiligo. Between 15 and $20 \%$ of patients have one or more familial first-degree relatives with vitiligo indicating the sporadic nature of occurrence of non-segmental vitiligo. Moreover, a $23 \%$ concordance of the disease has been noted in monozygotic twins (Spritz 2013). This signifies the penetrance of the genetic predisposition to vitiligo and heritability of vitiligo-associated genes (Alikhan et al. 2011). Identifying vitiligo susceptibility genes and characterizing the stress responses against trigger events that delineate autoimmune components causing disease progression would impel towards significant progress in understanding vitiligo etiology. Various studies have identified that the majority of the susceptible gene variants inculpates modulation of the immune system along with genes linked with melanocytes proliferation and migration (Zhang and Xiang 2014). It is the modulation and alteration exerted by specific genetic variations and their interactions that predispose inflammatory responses targeting melanocyte death owing to the onset of vitiligo.

Genetic studies have identified a majority of the genes that are associated with vitiligo to be a subset of immune regulatory genes concerning immuno-modulatory functions. However, a small number of genes have been identified to be linked to functions involving the regulation of melanocytes growth and proliferation, thus supporting immune regulatory mechanisms as the process that manifest vitiligo. The functional role of the many identified susceptibility loci in the pathogenesis of vitiligo is not known yet which remarkably overlaps with the genes that influence other autoimmune disorders underlining our incomplete knowledge of the disease mechanism (Shen et al. 2016). Genetic mutations that have the potential to modulate both the innate and adaptive immune responses increase the risk for vitiligo susceptibility. Table 2 lists the genetic variants that have been reported to be associated with vitiligo susceptibility. Mutations in several genes in the HLA class I and II regions encoding MHC have been discovered to be associated with vitiligo (Spritz 2012; Shen et al. 2016). MHCs are responsible for the processing and presentation 
Table 2 Vitiligo susceptible genetic variants

\begin{tabular}{|c|c|c|c|}
\hline Gene & Locus & Protein & Role \\
\hline $\mathrm{BACH} 2$ & $6 q 15$ & BTB domain and CNC homolog 2 & B cell regulator \\
\hline BTNL2 & $6 p 21.32$ & Butyrophilin-like 2 & $\mathrm{~T}$ cell regulator \\
\hline CASP7 & $10 q 25.3$ & Caspase 7 & Apoptosis \\
\hline CAT & $11 \mathrm{p} 13$ & Catalase & Oxidative stress regulator \\
\hline CCR6 & $6 q 27$ & C-C motif chemokine receptor 6 & B cell, T cell and dendritic cell regulator \\
\hline CD44 & $11 p 13$ & CD44 antigen & $\mathrm{T}$ cell regulator \\
\hline CD80 & $3 q 13.33$ & T-lymphocyte activation antigen CD80 & $\begin{array}{l}\text { T cell priming by B cells, T cells and dendritic } \\
\text { cells }\end{array}$ \\
\hline CLNK & $4 \mathrm{p} 16.1$ & Cytokine-dependent hematopoietic cell linker & Positive regulator of mast cells \\
\hline C1QTNF6 & $22 q 12.3$ & $\begin{array}{l}\text { Complement C1q tumor necrosis factor-related protein } \\
6\end{array}$ & Immune response-induced apoptosis \\
\hline CTLA4 & $2 q 33.2$ & Cytotoxic T-lymphocyte protein 4 & T cell inhibition \\
\hline CXCR5 & $11 \mathrm{q} 23.3$ & $\mathrm{C}-\mathrm{X}-\mathrm{C}$ motif chemokine receptor 5 & B cell activity \\
\hline FGFR1OP & $6 q 27$ & Fibroblast growth factor receptor 1 oncogene partner & Cell growth and proliferation \\
\hline FOXP3 & Xp11.23 & Forkhead box protein P1 & $\mathrm{T}$ cell activity and development \\
\hline GSTP1 & $11 q 13.2$ & Glutathione S-transferase Pi 1 & Oxidative stress regulator \\
\hline GZMB & $14 q 12$ & Granzyme B & Cytotoxic T lymphocyte-mediated death \\
\hline HLA-A, HLA-B and HLA-C & $6 \mathrm{p} 22.1$ & Human leukocyte antigen A, B and C & Peptide antigen presentation \\
\hline $\begin{array}{l}\text { HLA-DQB1 and HLA- } \\
\text { DRB1 }\end{array}$ & $6 p 21.32$ & Human leukocyte antigen DQB1 and DRB1 & Peptide antigen presentation \\
\hline$|\mathrm{F}| \mathrm{H} 1$ & $2 q 24.2$ & $\begin{array}{l}\text { Interferon-induced helicase C domain containing } \\
\text { protein } 1\end{array}$ & Innate immune activity \\
\hline IFNAR1 & $21 q 22.11$ & Interferon alpha and beta receptor subunit 1 & $\mathrm{~T}$ cell signaling \\
\hline IKZF4 & $12 q 13.2$ & Zinc finger protein Eos & $\mathrm{T}$ cell regulator \\
\hline IL2RA & 10p15.1 & Interleukin-2 receptor subunit alpha & Interleukin 2-dependent T cell activation \\
\hline LTA & $6 p 21.33$ & Lymphotoxin alpha & Immune response-induced apoptosis \\
\hline MC1R & $16 q 24.3$ & Melanocortin 1 receptor & Melanogenesis regulator \\
\hline MTHFR & 1 p36.22 & Methylenetetrahydrofolate reductase & Oxidative stress regulator \\
\hline NLRP1 & $17 p 13.2$ & NLR family pyrin domain containing 1 & $\begin{array}{l}\text { Innate immune } \\
\text { Activity }\end{array}$ \\
\hline OCA2 & $15 q 12-13-1$ & P protein & Melanosomal transporter \\
\hline PMEL & $12 q 13.2$ & Premelanosome protein & Melanosomes regulator \\
\hline PTPN22 & $1 \mathrm{p} 13.2$ & Tyrosine-protein phosphatase non-receptor type 22 & $\mathrm{~T}$ cell signaling \\
\hline RERE & $1 \mathrm{p} 36.23$ & Arginine-glutamic acid dipeptide repeats protein & Lymphoid co-repressor \\
\hline $\mathrm{SH} 2 \mathrm{~B} 3$ & $12 q 24.12$ & SH2B adaptor protein 3 & $\mathrm{~T}$ cell signaling \\
\hline SLA & $8 q 24.22$ & Src-like adaptor & T cell signaling \\
\hline SOD2 and SOD3 & $\begin{array}{l}6 \mathrm{q} 25.3 \\
4 \mathrm{p} 15.2\end{array}$ & Superoxide dismutase 2 and 3 & Oxidative stress regulator \\
\hline TICAM1 & 19p13.3 & Toll-like receptor adaptor molecule 1 & Innate immune activity \\
\hline TLR2 and TLR4 & $\begin{array}{l}4 q 31.3 \\
9 q 33.1\end{array}$ & Toll-like receptor 2 and 4 & Innate immune activity \\
\hline TNF & $6 p 21.33$ & Tumor necrosis factor & Cell proliferation, differentiation and apoptosis \\
\hline TYR & $11 \mathrm{q} 14.3$ & Tyrosinase & Melanogenesis regulator \\
\hline UBASH3A & $21 \mathrm{q} 22.3$ & Ubiquitin associated and $\mathrm{SH} 3$ domain containing $\mathrm{A}$ & T cell signaling \\
\hline ZMIM1 & $10 q 22.3$ & Zinc finger MIZ-type containing 1 & Transcriptional co-activator \\
\hline
\end{tabular}


of antigens and are associated with autoimmune diseases in the incidence of derailed recognition of self-antigens. Failure of the immune system to recognize self-antigens leads to the development of autoreactive $\mathrm{T}$ cells that eventually restrict the formation of an efficient regulatory $\mathrm{T}$ cell (Treg) population (Simmonds and Gough 2007). Also, genetic variants of CTLA4 that are involved in T cell inhibition have been implicated to have a role in vitiligo pathogenesis (Birlea et al. 2009). Genome-wide association studies (GWAS) have found alteration in the genes that encode for factors involved in $\mathrm{B}$ cell regulation (BACH2), $\mathrm{T}$ cell development and priming (CD44, CD80), T cell receptor signaling (SLA, PTPN22, UBASH3A, CLNK, IFNAR1), and T cell activation (IKZF4, IL2RA, BTNL2, FOXP3) to be associated with vitiligo. Altered expression of the genes that regulate the innate immune response (IFIH1, NLRP1, TICAM1, TLR) and chemokine or cytokine receptors (CXCR5, CCR6, SH2B3) have also been found to be associated with vitiligo (Jin et al. 2012; Karaca et al. 2013; Shen et al. 2016).

Polymorphisms in non-immune-related genes have also been identified as risk factors. The melanocyte-specific genes (TYR, PMEL, MC1R, OCA2) encode for proteins or enzymes that participate in melanin production, and the ZMIZI gene is involved in the regulation of melanocyte development and survival (Sun et al. 2014). Genetic variants of these genes may serve as $\mathrm{T}$ cell antigens (autoantigens) which facilitates the initiation of an anti-melanocyte immune response contributing to cellular stress and melanocyte damage resulting in the development of vitiligo (Jin et al. 2010; Spritz 2013). Variation in the genes FGFR1OP, RERE, and CASP7 which have a central role in apoptosis along with GZMB, C1QTNF6, LTA, and TNF which are involved in immune response-induced apoptosis has also been linked with vitiligo (Lee and Bae 2015; Shen et al. 2016). Recently, an elevated plasma level of homocysteine has been described in patients with vitiligo which is associated with the polymorphic expression of MTHFR. MTHFR regulates homocysteine levels in the plasma, and an increased expression renders higher susceptibility to oxidative stress thereby contributing to the damage of melanocytes in vitiligo patients (Chen et al. 2014). Additionally, the genes GSTP1, SOD, and CAT are all broadly expressed in defense against oxidative stress wherein altered expression of these genes impairs their defense mechanisms contributing to ROS-induced melanocyte death in vitiligo patients (Wood et al. 2008; Liu et al. 2009; Laddha et al. 2013).

\section{Scope of network-interaction studies and polypharmacological studies in understanding vitiligo disease module}

Network science and analysis involve systemic cataloging of molecular interactions that offer unforeseen perspective prospects to understand and analyze the internal cellular organization and the interconnections between disease-related genes and functional proteins. Network-based studies facilitate in elucidating the extensive complex cellular networks presenting an ideal framework of their cellular organization. Protein-protein interaction networks composed of multiple nodes connected by edges accommodate better estimation of network statistics contributing to a comprehensive assistance in discerning the cellular organizing principles and molecular mechanisms that dictate the manifestation of a disease cycle (Barabasi and Oltvai 2004). This is particularly propitious when interpreting polygenic disorders having intricate patterns. Protein-protein interactions are of prime importance for various cellular and regulatory processes. Genetic variation alters or damages protein structure inciting disruption in protein-protein interactions constituting the pretext of disease development. These interaction networks usually consist of a few essential nodes (called hubs) that show maximum interaction to a large number of neighboring nodes. According to the phenomena of the centrality-lethality rule, the identification of such hub proteins and their inhibition may be lethal for the network. The magnitude of the change in structure caused by the removal of a node in a network determines the relative importance of the node in the network. Removal of such structurally critical nodes (hubs) in a network is widely believed to reflect the significance of the network architecture to better ascertain the network functionality (He and Zhang 2006). These hubs in a protein-protein interaction network may represent potential drug candidates. Since drug discovery and development is a complicated and expensive process, polypharmacology has emerged as the next paradigm of drug discovery. The transformation in the philosophy of current drug designing from one drug-one target to multiple targets of a single drug incorporates polypharmacological analysis that intends to discover the unknown targets for the existing drugs (Yildirim et al. 2007). Polypharmacology-based integrated systems biology approaches along with computational modeling, pharmacological, and clinical studies are productive for identification of novel molecular determinants essential in drug discovery and development. It also aids in unraveling the understanding of the significant impact of a new drug on complex human diseases (Rahman et al. 2018). A drug showing connections with multiple nodes (targets) in a network implies its high efficacious potential to control or inhibit the function of the particular target that is detrimental for regulatory pathways (Boran and Iyengar 2010). The identification of such hub proteins in vitiligo disease network together with polypharmacological studies will serve as an effective practical approach towards improved therapeutic interventions for better vitiligo management. 


\section{Conclusions}

Cellular and molecular genetics studies have improved our knowledge on disease pathogenesis opening possibilities for new targeted therapies. An understanding of the mechanisms leading to melanocyte degeneration and autoimmunity and how melanocytes interact with their surroundings may help to unravel the rationale for the lack of melanocyte regeneration in vitiligo. Also, profiling the biological mediators of disease mechanisms might identify new therapeutic targets that may arrest disease progression and promote cell regeneration thereby stimulating repigmentation in the affected areas. Improved therapeutic and diagnostic modalities would substantially increase the compliance and satisfaction of patients. Also, the association of vitiligo with other diseases indicates that the knowledge of vitiligo pathogenesis could be of importance other than the skin as well.

\section{Abbreviations}

CMV: Cytomegalovirus; GWAS: Genome-wide association studies; HBV: Hepatitis B virus; HIV: Human immunodeficiency virus; HLA: Human leukocyte antigen; HO1: Heme oxygenase 1; MART-1: Melanoma antigen recognized by T cells 1; MHC: Major histocompatibility complex; NBUVB: Narrow-band UVB; NLRP1: NLR family pyrin domain containing 1; NPY: Neuropeptide Y; PDGFRB: Platelet-derived growth factor receptor $\beta$; ROS: Reactive oxygen species; SIRT1: Sirtuin 1; TRP: Tyrosinase; UTR: Untranslated region; XBP1: X-box binding protein

\section{Acknowledgements}

Not applicable.

\section{Funding}

This work was supported by the Department of Biotechnology, Government of India [No.BT/PR5402/BID/7/508/2012].

\section{Availability of data and materials}

Not applicable.

\section{Authors' contributions}

$\mathrm{YH}$ conceived and designed the study. RR and $\mathrm{YH}$ prepared the manuscript. Both the authors read and approved the final manuscript.

\section{Ethics approval and consent to participate}

Not applicable.

\section{Consent for publication}

Not applicable.

\section{Competing interests}

The authors declare that they have no competing interests.

\section{Publisher's Note}

Springer Nature remains neutral with regard to jurisdictional claims in published maps and institutional affiliations.

Received: 4 January 2018 Accepted: 27 June 2018

Published online: 15 August 2018

\section{References}

Akbayir N, Gokdemir G, Mansur T, Sokmen M, Gündüz S, Alkim C, Barutcuoglu B, Erdem $L$. Is there any relationship between hepatitis $C$ virus and vitiligo? J Clin Gastroenterol. 2004;38:815-7.

Akcan Y, Kavak A, Sertbas Y, Olut Al, Korkut E, Bicik Z, Kisacik B. The low seropositivity of hepatitis B virus in vitiligo patients. J Eur Acad Dermatol Venereol. 2006;20(1):110-1.

AlGhamdi KM, Kumar A. Depigmentation therapies for normal skin in vitiligo universalis. J Eur Acad Dermatol Venereol. 2011;25(7):749-57.
Alikhan A, Felsten LM, Daly M, Petronic-Rosic V. Vitiligo: a comprehensive overview Part, I Introduction, epidemiology, quality of life, diagnosis, differential diagnosis, associations, histopathology, etiology, and work-up. J Am Acad Dermatol. 2011;65:473-91.

Barabasi AL, Oltvai ZN. Network biology: understanding the cell's functional organization. Nat Rev Genet. 2004;5(2):101-13.

Bellei B, Pitisci A, Ottaviani M, Ludovici M, Cota C, Luzi F, Dell'Anna ML, Picardo M. Vitiligo: a possible model of degenerative diseases. PLoS One. 2013;8(3):e59782.

Ben Ahmed M, Zaraa I, Rekik R, Elbeldi-Ferchiou A, Kourda N, Belhadj Hmida N, Abdeladhim M, Karoui O, Ben Osman A, Mokni M, Louzir H. Functional defects of peripheral regulatory T lymphocytes in patients with progressive vitiligo. Pigment Cell Melanoma Res. 2012;25(1):99-109.

Bhatnagar A, Kanwar AJ, Parsad D, De D. Comparison of systemic PUVA and NBUVB in the treatment of vitiligo: an open prospective study. J Eur Acad Dermatol Venereol. 2007;21(5):638-42.

Birlea SA, LaBerge GS, Procopciuc LM, Fain PR, Spritz RA. CTLA4 and generalized vitiligo: two genetic association studies and a meta-analysis of published data. Pigment Cell Melanoma Res. 2009;22(2):230-4

Boisseau-Garsaud AM, Garsaud P, Cales-Quist D, Helenon R, Queneherve C, Claire RC. Epidemiology of vitiligo in the French West Indies (Isle of Martinique). Int J Dermatol. 2000;39(1):18-20.

Boran AD, lyengar R. Systems approaches to polypharmacology and drug discovery. Curr Opin Drug Discov Devel. 2010;13(3):297.

Chang CL, Au LC, Huang SW, Fai Kwok C, Ho LT, Juan CC. Insulin up-regulates heme oxygenase-1 expression in 3T3-L1 adipocytes via PI3-kinase- and PKCdependent pathways and heme oxygenase-1-associated microRNA downregulation. Endocrinology. 2011;152:384-93.

Chen JX, Shi Q, Wang XW, Guo S, Dai W, Li K, Song P, Wei C, Wang G, Li CY, Gao TW. Genetic polymorphisms in the methylenetetrahydrofolate reductase gene (MTHFR) and risk of vitiligo in Han Chinese populations: a genotypephenotype correlation study. Br J Dermatol. 2014;170(5):1092-9.

Dell'Anna ML, Picardo M. A review and a new hypothesis for non-immunological pathogenetic mechanisms in vitiligo. Pigment Cell Melanoma Res. 2006;19(5):406-11.

Ezzedine K, Diallo A, Leaute-Labreze C, Seneschal J, Boniface K, Cario-Andre M, Prey S, Ballanger F, Boralevi F, Jouary T, Mossalayi D. Pre-vs. post-pubertal onset of vitiligo: multivariate analysis indicates atopic diathesis association in pre-pubertal onset vitiligo. Br J Dermatol. 2012a;167(3):490-5.

Ezzedine K, Gauthier Y, Leaute-Labreze C, Marquez S, Bouchtnei S, Jouary T, Taieb A. Segmental vitiligo associated with generalized vitiligo (mixed vitiligo): a retrospective case series of 19 patients. J Am Acad Dermatol. 2011;65(5):965-71.

Ezzedine K, Lim HW, Suzuki T, Katayama I, Hamzavi I, Lan CC, Goh BK, Anbar T, Silva de Castro C, Lee AY, Parsad D. Revised classification/nomenclature of vitiligo and related issues: the vitiligo global issues consensus conference. Pigment Cell Melanoma Res. 2012b;25(3)

Gauthier Y, Cario-Andre M, Lepreux S, Pain C, Taieb A. Melanocyte detachment after skin friction in non lesional skin of patients with generalized vitiligo. $\mathrm{Br} J$ Dermatol. 2003;148(1):95-101.

Geel N, Bosma S, Boone B, Speeckaert R. Classification of segmental vitiligo on the trunk. Br J Dermatol. 2014;170(2):322-7.

Gey A, Diallo A, Seneschal J, Leaute-Labreze C, Boralevi F, Jouary T, Taieb A, Ezzedine K. Autoimmune thyroid disease in vitiligo: multivariate analysis indicates intricate pathomechanisms. Br J Dermatol. 2013;168(4):756-61.

Gupta V, Sreenivas V, Mehta M, Khaitan BK, Ramam M. Measurement properties of the Vitiligo impact Scale-22 (VIS-22), a vitiligo-specific quality-of-life instrument. Br J Dermatol. 2014;171(5):1084-90.

Halder RM. Childhood vitiligo. Clin Dermatol. 1997;15:899-906.

He $X$, Zhang J. Why do hubs tend to be essential in protein networks? PLoS Genet. 2006;2(6):e88.

Hossain C, Porto DA, Hamzavi I, Lim HW. Camouflaging agents for vitiligo patients. J Drugs Dermatol. 2016;15(4):384-7.

Howitz J, Brodthagen H, Schwartz M, Thomsen K. Prevalence of vitiligo: epidemiological survey on the Isle of Bornholm, Denmark. Arch Dermatol. 1977;113(1):47-52.

Jablonski NG, Chaplin G. Human skin pigmentation as an adaptation to UV radiation. Proc Natl Acad Sci USA. 2010;107(Supplement 2):8962-8.

Jiang L, Cheng Z, Qiu S, Que Z, Bao W, Jiang C, Zou F, Liu P, Liu J. Altered let-7 expression in myasthenia gravis and let-7c mediated regulation of IL-10 by directly targeting IL-10 in Jurkat cells. Int Immunopharmacol. 2012;14:217-23.

Jimbow K, Chen H, Park JS, Thomas PD. Increased sensitivity of melanocytes to oxidative stress and abnormal expression of tyrosinase-related protein in vitiligo. Br J Dermatol. 2001;144(1):55-65. 
Jin Y, Birlea SA, Fain PR, Ferrara TM, Ben S, Riccardi SL, Cole JB, Gowan K, Holland PJ, Bennett DC, Luiten RM. Genome-wide association analyses identify 13 new susceptibility loci for generalized vitiligo. Nat Genet. 2012;44(6):676.

Jin Y, Birlea SA, Fain PR, Gowan K, Riccardi SL, Holland PJ, Mailloux CM, Sufit AJ, Hutton SM, Amadi-Myers A, Bennett DC. Variant of TYR and autoimmunity susceptibility loci in generalized vitiligo. N Engl J Med. 2010;362:1686-97.

Karaca N, Ozturk G, Gerceker BT, Turkmen M, Berdeli A. TLR2 and TLR4 gene polymorphisms in Turkish vitiligo patients. J Eur Acad Dermatol Venereol. 2013;27(1):e85-90.

Kasumagic-Halilovic E, Ovcina-Kurtovic N, Jukic T, Karamehic J, Begovic B, Samardzic S. Vitiligo and autoimmunity. Med Arch. 2013;67(2):91.

Kasumagic-Halilovic E, Prohic A, Begovic B, Ovcina-Kurtovic N. Association between vitiligo and thyroid autoimmunity. J Thyroid Res. 2011;3:938257.

Kemp EH, Emhemad S, Akhtar S, Watson PF, Gawkrodger DJ, Weetman AP. Autoantibodies against tyrosine hydroxylase in patients with non-segmental (generalised) vitiligo. Exp Dermatol. 2011;20:35-40.

Kim DY, Oh SH, Hann SK. Classification of segmental vitiligo on the face: clues for prognosis. Br J Dermatol. 2011;164(5):1004-9.

Krüger C, Schallreuter KU. A review of the worldwide prevalence of vitiligo in children/adolescents and adults. Int J Dermatol. 2012;51(10):1206-12.

Kruger C, Schallreuter KU. Cumulative life course impairment in vitiligo. Curr Probl Dermatol. 2013;44:102-17.

Laddha NC, Dwivedi M, Gani AR, Shajil EM, Begum R. Involvement of superoxide dismutase isoenzymes and their genetic variants in progression of and higher susceptibility to vitiligo. Free Radic Biol Med. 2013;65:1110-25.

Lang KS, Muhm A, Moris A, Stevanovic S, Rammensee HG, Caroli CC, Wernet D, Schittek B, Knauss-Scherwitz E, Garbe C. HLA-A2 restricted, melanocytespecific CD8+ T lymphocytes detected in vitiligo patients are related to disease activity and are predominantly directed against MelanA/MART1. J Invest Dermatol. 2001;116(6):891-7.

Lazarova R, Hristakieva E, Lazarov N, Shani J. Vitiligo-related neuropeptides in nerve fibers of the skin. Arch Physiol Biochem. 2000;108(3):262-7.

Le Poole IC, Das PK, van den Wijngaard RM, Bos JD, Westerhof W. Review of the etiopathomechanism of vitiligo: a convergence theory. Exp Dermatol. 1993; (4):145-53.

Le Poole IC, Luiten RM. Autoimmune etiology of generalized vitiligo. Curr Dir Autoimmun. 2008;10:227-43.

Le Poole IC, van den Wijngaard RM, Westerhof W, Das PK. Tenascin is overexpressed in vitiligo lesional skin and inhibits melanocyte adhesion. Br J Dermatol. 1997;137(2):171-8.

Lee YH, Bae SC. Associations between TNF-a polymorphisms and susceptibility to rheumatoid arthritis and vitiligo: a meta-analysis. Genet Mol Res. 2015;14(2):5548-9.

Leone G, Paro VA. Effect of an antioxydant cream versus placebo in patients with vitiligo in association with excimer laser: a pilot randomized, investigator-blinded, and half-side comparison trial. G Ital Dermatol Venereol. 2015;150(4):461-6.

Levandowski CB, Mailloux CM, Ferrara TM, Gowan K, Ben S, Jin Y, McFann KK, Holland PJ, Fain PR, Dinarello CA, Spritz RA. NLRP1 haplotypes associated with vitiligo and autoimmunity increase interleukin-1 $\beta$ processing via the NLRP1 inflammasome. Proc Natl Acad Sci U S A. 2013;110(8):2952-6.

Lili Y, Yi W, Ji Y, Yue S, Weimin S, Ming L. Global activation of CD8+ cytotoxic T lymphocytes correlates with an impairment in regulatory $T$ cells in patients with generalized vitiligo. PLoS One. 2012;7:e37513.

Lilly E, Lu PD, Borovicka JH, Victorson D, Kwasny MJ, West DP, Kundu RV. Development and validation of a vitiligo-specific quality-of-life instrument (VitiQoL). J Am Acad Dermatol. 2013;69(1):e11-8.

Liu L, Li C, Gao J, et al. Genetic polymorphisms of glutathione S-transferase and risk of vitiligo in the Chinese population. J Invest Dermatol. 2009;129(11):2646-52.

Mansuri MS, Singh M, Begum R. miRNA signatures and transcriptional regulation of their target genes in vitiligo. J Dermatol Sci. 2016;84(1):50-8.

Mansuri MS, Singh M, Dwivedi M, Laddha NC, Marfatia YS, Begum R. MicroRNA profiling reveals differentially expressed microRNA signatures from the skin of patients with nonsegmental vitiligo. Br J Dermatol. 2014;171:1263-7.

Maresca V, Roccella M, Roccella F, Camera E, Del Porto G, Passi S, Grammatico P, Picardo M. Increased sensitivity to peroxidative agents as a possible pathogenic factor of melanocyte damage in vitiligo. J Investig Dermatol. 1997;109(3):310-3.

Marie J, Kovacs D, Pain C, Jouary T, Cota C, Vergier B, Picardo M, Taieb A, Ezzedine K, Cario-Andre M. Inflammasome activation and vitiligo/ nonsegmental vitiligo progression. Br J Dermatol. 2014;170(4):816-23.
Niamba P, Traore A, Taieb A. Vitiligo in a black patient associated with HIV infection and repigmentation under antiretroviral therapy. Ann Dermatol Venereol. 2007; 134:272.

Nicolaidou E, Antoniou C, Miniati A, Lagogianni E, Matekovits A, Stratigos A, Katsambas A. Childhood- and later-onset vitiligo have diverse epidemiologic and clinical characteristics. J Am Acad Dermatol. 2012;66:954-8.

Njoo MD, Spuls PI, Bos JT, Westerhof W, Bossuyt PM. Nonsurgical repigmentation therapies in vitiligo: meta-analysis of the literature. Arch Dermatol. 1998; 134(12):1532-40

Nordal EJ, Guleng GE, Rönnevig JR. Treatment of vitiligo with narrowband-UVB (TL01) combined with tacrolimus ointment (0.1\%) vs. placebo ointment, a randomized right/left double-blind comparative study. J Eur Acad Dermatol Venereol. 2011;25(12):1440-3.

Ormerod AD. Topical tacrolimus and pimecrolimus and the risk of cancer: how much cause for concern? Br J Dermatol. 2005;153(4):701-5.

Oyarbide-Valencia K, van den Boorn JG, Denman CJ, Li M, Carlson JM, Hernandez C, Nishimura MI, Das PK, Luiten RM, Le Poole IC. Therapeutic implications of autoimmune vitiligo T cells. Autoimmun Rev. 2006;5(7):486-92.

Paradisi A, Tabolli S, Didona B, Sobrino L, Russo N, Abeni D. Markedly reduced incidence of melanoma and nonmelanoma skin cancer in a nonconcurrent cohort of 10,040 patients with vitiligo. J Am Acad Dermatol. 2014;71(6):1110-6.

Parsad D, Dogra S, Kanwar AJ. Quality of life in patients with vitiligo. Health Qual Life Outcomes. 2013;1:58.

Picardo M, Dell'Anna ML, Ezzedine K, Hamzavi I, Harris JE, Parsad D, Taieb A. Vitiligo. Nat Rev Dis Primers. 2015;1:15011.

Rahman R, Sharma I, Gahlot LK, Hasija Y. DermaGene and VitmiRS: a comprehensive systems analysis of genetic dermatological disorders. Biomed Dermatol. 2018;2(1):18.

Ratsep R, Kingo K, Karelson M, Reimann E, Raud K, Silm H, Vasar E, Koks S. Gene expression study of IL10 family genes in vitiligo skin biopsies, peripheral blood mononuclear cells and sera. Br J Dermatol. 2008;159:1275-81.

Rezaei N, Gavalas NG, Weetman AP, Kemp EH. Autoimmunity as an aetiological factor in vitiligo. J Eur Acad Dermatol Venereol. 2007;21(7):865-76.

Ruksha TG, Komina AV, Palkina NV. MicroRNA in skin diseases. Eur J Dermatol. 2017;27(4):343-52.

Sahmatova L, Tankov S, Prans E, Aab A, Hermann H, Reemann P, Pihlap M, Karelson M, Abram K, Kisand K, Kingo K. MicroRNA-155 is dysregulated in the skin of patients with vitiligo and inhibits melanogenesis-associated genes in melanocytes and keratinocytes. Acta Derm Venereol. 2016;96(6):742-8.

Salzes C, Abadie S, Seneschal J, Whitton M, Meurant JM, Jouary T, Ballanger F, Boralevi F, Taieb A, Taieb C, Ezzedine K. The Vitiligo Impact Patient Scale (VIPS): development and validation of a vitiligo burden assessment tool. J Investig Dermatol. 2016;136(1):52-8.

Saunders LR, Sharma AD, Tawney J, Nakagawa M, Okita K, Yamanaka S, Willenbring H, Verdin E. miRNAs regulate SIRT1 expression during mouse embryonic stem cell differentiation and in adult mouse tissues. Aging (Albany NY). 2010;2:415-31.

Sehgal VN, Srivastava G. Vitiligo: compendium of clinico-epidemiological features. Indian J Dermatol Venereol Leprol. 2007;73(3):149.

Senol A, Yucelten AD, Ay P. Development of a quality of life scale for vitiligo. Dermatology. 2013;226(2):185-90.

Shah R, Hunt J, Webb TL, Thompson AR. Starting to develop self-help for social anxiety associated with vitiligo: using clinical significance to measure the potential effectiveness of enhanced psychological self-help. Br J Derm. 2014;171:332-7.

Shen C, Gao J, Sheng Y, Dou J, Zhou F, Zheng X, Ko R, Tang X, Zhu C, Yin X, Sun L. Genetic susceptibility to vitiligo: GWAS approaches for identifying vitiligo susceptibility genes and loci. Front Genet. 2016;7:3.

Shi YL, Weiland M, Li J, Hamzavi I, Henderson M, Huggins RH, Mahmoud BH, Agbai O, Mi X, Dong Z, Lim HW. MicroRNA expression profiling identifies potential serum biomarkers for non-segmental vitiligo. Pigment Cell Melanoma Res. 2013;26(3):418-21.

Simmonds MJ, Gough SC. The HLA region and autoimmune disease: associations and mechanisms of action. Curr Genomics. 2007;8(7):453-65.

Sitek JC, Loeb M, Ronnevig JR. Narrowband UVB therapy for vitiligo: does the repigmentation last? J Eur Acad Dermatol Venereol. 2007;21(7):891-6.

Spritz RA. The genetics of generalized vitiligo: autoimmune pathways and an inverse relationship with malignant melanoma. Genom Med. 2010;2(10):78.

Spritz RA. Six decades of vitiligo genetics: genome-wide studies provide insights into autoimmune pathogenesis. J Investig Dermatol. 2012;132(2):268-73.

Spritz RA. Modern vitiligo genetics sheds new light on an ancient disease. J Dermatol. 2013;40(5):310-8. 
Sravani PV, Babu NK, Gopal KV, Rao GR, Rao AR, Moorthy B, Rao TR. Determination of oxidative stress in vitiligo by measuring superoxide dismutase and catalase levels in vitiliginous and non-vitiliginous skin. Indian J Dermatol Venereol Leprol. 2009:75(3):268.

Strassner JP, Harris JE. Understanding mechanisms of autoimmunity through translational research in vitiligo. Curr Opin Immunol. 2016;43:81-8.

Sun Y, Zuo X, Zheng X, Zhou F, Liang B, Liu H, Chang R, Gao J, Sheng Y, Cui H, Wang W. A comprehensive association analysis confirms ZMIZ1 to be a susceptibility gene for vitiligo in Chinese population. J Med Genet. 2014; 51(5):345-53.

Taher ZA, Lauzon G, Maguiness S, Dytoc MT. Analysis of interleukin-10 levels in lesions of vitiligo following treatment with topical tacrolimus. Br J Dermatol. 2009;161(3):654-9.

Taieb A. Vitiligo as an inflammatory skin disorder: a therapeutic perspective. Pigment Cell Melanoma Res. 2012;25(1):9-13.

Teovska Mitrevska N, Eleftheriadou V, Guarneri F. Quality of life in vitiligo patients. Dermatol Ther. 2012;25(Suppl. 1):S28-31.

Toker SC, Sarycaoglu H, Karadogan SK, Mistik R, Baskan EB, Tunaly S. Is there any relation between vitiligo and cytomegalovirus? J Eur Acad Dermatol Venereol. 2007;21:141-2.

Van Den Boorn JG, Konijnenberg D, Dellemijn TA, Van Der Veen JW, Bos JD, Melief CJ, Vyth-Dreese FA, Luiten RM. Autoimmune destruction of skin melanocytes by perilesional T cells from vitiligo patients. J Invest Dermatol. 2009;129(9):2220-32.

van Geel N, Speeckaert R, De Wolf J, Bracke S, Chevolet I, Brochez L, Lambert J. Clinical significance of Koebner phenomenon in vitiligo. Br J Dermatol. 2012; 167(5):1017-24

van Geel N, Wallaeys E, Goh BK, De Mil MA, Lambert J. Long-term results of noncultured epidermal cellular grafting in vitiligo, halo naevi, piebaldism and naevus depigmentosus. Br J Dermatol. 2010;163(6):1186-93.

Wagner RY, Luciani F, Cario-Andre M, Rubod A, Petit V, Benzekri L, Ezzedine K, Lepreux S, Steingrimsson E, Taieb A, Gauthier Y. Altered E-cadherin levels and distribution in melanocytes precede clinical manifestations of vitiligo. J Investig Dermatol. 2015;135(7):1810-9.

Wang X, Du J, Wang T, Zhou C, Shen Y, Ding X, Tian S, Liu Y, Peng G, Xue S, Zhou J, Wang R, Meng X, Pei G, Bai Y, Liu Q, Li H, Zhang J. Prevalence and clinical profile of vitiligo in China: a community-based study in six cities. Acta Derm Venereol. 2013;93(1):62-5.

Wood JM, Gibbons NC, Chavan B, Schallreuter KU. Computer simulation of heterogeneous single nucleotide polymorphisms in the catalase gene indicates structural changes in the enzyme active site, NADPH-binding and tetramerization domains: a genetic predisposition for an altered catalase in patients with vitiligo? Exp Dermatol. 2008;17(4):366-71.

Yildirim MA, Goh Kl, Cusick ME, Barabasi AL, Vidal M. Drug-target network. Nat Biotechnol. 2007;25(10):1119-26.

Zhang Z, Xiang LF. Genetic susceptibility to vitiligo: recent progress from genome-wide association studies. Dermatol Sin. 2014;32(4):225-32.

\section{Ready to submit your research? Choose BMC and benefit from:}

- fast, convenient online submission

- thorough peer review by experienced researchers in your field

- rapid publication on acceptance

- support for research data, including large and complex data types

- gold Open Access which fosters wider collaboration and increased citations

- maximum visibility for your research: over $100 \mathrm{M}$ website views per year

At BMC, research is always in progress.

Learn more biomedcentral.com/submissions 